\title{
Effective dose from radiopharmaceuticals
}

\author{
Lennart Johansson', Sören Mattson², Bertil Nosslin², Sigrid Leide-Svegborn² \\ 1 Department of Radiation Physics, Umeá University Hospital, S-901 85 Umeå, Sweden \\ ${ }^{2}$ Department of Radiation Physics, Malmö University Hospital, S-214 01 Malmö, Sweden
}

Eur J Nucl Med (1993) 19:933-938

An unfortunate error in the calculation program resulted in too high effective dose values for a minor fraction of the substances listed. For the iron isotopes, ${ }^{51} \mathrm{Cr}$ and ${ }^{99} \mathrm{~m}$ Tc labelled erythrocytes, ${ }^{99 \mathrm{~m}} \mathrm{Tc}$ and ${ }^{123} \mathrm{I}$ labelled fibrinogen and for ${ }^{99 \mathrm{~m}} \mathrm{Tc}$ HSA, the error can be considered as significant in relation to the overall uncertainty of the effective dose estimates. The correct value for these substances is therefore given below.
Table 1. Effective dose per unit administered activity

\begin{tabular}{lll}
\hline Radionuclide & Substance & $\begin{array}{l}\mathrm{E} \\
(\mathrm{mSv} / \mathrm{MBq})\end{array}$ \\
\hline${ }^{51} \mathrm{Cr}$ & Cr-labelled erythrocytes & $1.7 \mathrm{E}-01$ \\
${ }^{52} \mathrm{Fe}$ & Iron & $1.1 \mathrm{E}+00$ \\
${ }^{52} \mathrm{Fe}$ & Iron (Oral administration) & $7.1 \mathrm{E}-01$ \\
${ }^{55} \mathrm{Fe}$ & Iron & $4.0 \mathrm{E}+00$ \\
${ }^{55} \mathrm{Fe}$ & Iron (Oral administration) & $4.2 \mathrm{E}-01$ \\
${ }^{59} \mathrm{Fe}$ & Iron & $1.0 \mathrm{E}+01$ \\
${ }^{59} \mathrm{Fe}$ & Iron (Oral administration) & $2.0 \mathrm{E}+00$ \\
${ }^{99 \mathrm{~m}} \mathrm{Tc}$ & Tc-labelled albumin (HSA) & $6.1 \mathrm{E}-03$ \\
${ }^{99} \mathrm{mc}$ & Tc-labelled fibrinogen & $6.2 \mathrm{E}-03$ \\
${ }^{99 \mathrm{~m}} \mathrm{Tc}$ & Tc-labelled erythrocytes & $6.6 \mathrm{E}-03$ \\
${ }^{123} \mathrm{I}$ & Iodine-labelled fibrinogen & $2.0 \mathrm{E}-02$ \\
\hline
\end{tabular}

